\title{
ON THE RELATION BETWEEN FOURIER AND LEONT'EV COEFFICIENTS WITH RESPECT TO SMIRNOV SPACES
}

\section{B. Forster}

UDC 517.5

Yu. Mel'nik showed that the Leont'ev coefficients $\kappa_{f}(\lambda)$ in the Dirichlet series $f \sim$ $\sum_{\lambda \in \Lambda} \kappa_{f}(\lambda) \frac{e^{\lambda}}{L^{\prime}(\lambda)}$ of a function $f \in E^{p}(D), 1<p<\infty$, are the Fourier coefficients of some function $F \in L^{p}([0,2 \pi])$ and that the first modulus of continuity of $F$ can be estimated by the first moduli and majorants in $f$. In the present paper, we extend his results to moduli of arbitrary order.

\section{Introduction}

Let $\bar{D}$ be a closed convex polygon with vertices $a_{1}, \ldots, a_{N}, N>2$, let $D$ be its open part, and let $\partial D=$ $\bar{D} \backslash D$ be the boundary of $D$. We assume that the origin belongs to $D$. As is customary, we denote by $E^{p}(D)$, $1<p<\infty$, the Banach space of all functions $f(z)$ analytic in $D$ and satisfying the condition

$$
\|f\|_{p}:=\sup _{n \in \mathbb{N}} \int_{\gamma_{n}}|f(z)|^{p}|d z|<\infty
$$

Here, $\left(\gamma_{n}\right)_{n \in \mathbb{N}}$ is a sequence of closed rectifiable Jordan contours $\gamma_{n} \subset D$ that converges to $\partial D$. The space $E^{p}(D)$ is called a Smirnov space.

Consider the quasipolynomial

$$
L(z)=\sum_{k=1}^{N} d_{k} e^{a_{k} z},
$$

where $d_{k} \in \mathbb{C} \backslash\{0\}$ and $a_{k}$ are the vertices of $D, k=1, \ldots, N$. Let $\Lambda=\left(\lambda_{m}\right)_{m \in \mathbb{N}}$ be its sequence of zeros. We can expand functions $f \in E^{p}(D)$ with respect to the family $\varepsilon(\Lambda):=\left(e^{\lambda_{m} z}\right)_{m \in \mathbb{N}}$ into a series of complex exponentials, namely, the so-called Dirichlet series

$$
f(z) \sim \sum_{\lambda_{m} \in \mathbb{N}} \kappa_{f}\left(\lambda_{m}\right) \frac{e^{\lambda_{m} z}}{L^{\prime}\left(\lambda_{m}\right)},
$$

where

Zentrum Mathematik, Technische Universität München, Germany. Published in Ukrains'kyi Matematychnyi Zhurnal, Vol. 56, No. 4, pp. 517-526, April, 2004. Original article submitted March 21, 2002. 


$$
\kappa_{f}\left(\lambda_{m}\right)=\sum_{k=1}^{N} d_{k} e^{a_{k} \lambda_{m}} \int_{a_{j}}^{a_{k}} f(\eta) e^{-\lambda_{m} \eta} d \eta
$$

The indexing of $\Lambda$ is chosen such that $\left(\left|\lambda_{m}\right|\right)_{m \in \mathbb{N}}$ is nondecreasing. The coefficients $\kappa_{f}\left(\lambda_{m}\right)$ are called Leont'ev coefficients. Many results on these series are due to Leont'ev [1]. Lewin and Ljubarskii showed in [2] that, for $p=2$, the family $\varepsilon(\Lambda)$ forms a Riesz basis of $E^{2}(D)$, and, hence, series (1) converges unconditionally in norm. In [3], Sedletskii proved that, for arbitrary $1<p<\infty$, the Dirichlet series (1) converges in norm since $\varepsilon(\Lambda)$ forms a Schauder basis in $E^{p}(D)$.

To estimate the rate of convergence of these series, Mel'nik studied the relation between Leont'ev coefficients and Fourier coefficients, since, for the latter, many results on approximation and rate of convergence of the Fourier series are well known (see, e.g., [4]). He showed that, under certain conditions, the Leont'ev coefficients of $f \in E^{p}(D)$ are the Fourier coefficients of some function $F \in L^{p}([0,2 \pi])$. He estimated the regularity of $F$ with first moduli of continuity. In Sec. 2, we state his results. Extending Mel'nik's Theorem 1 to moduli of smoothness of arbitrary order, we obtain Theorem 2 in Sec. 3. The last section contains the respective proof.

\section{Mel'nik's Results}

In [5] and [6], Mel'nik considered the relation of the Leont'ev coefficients of $f \in E^{p}(D)$ to the Fourier coefficients of some suited function $F \in L^{p}([0,2 \pi])$ for the first moduli of continuity. His first step was the reduction of the integral in (2) to a Fourier transform:

\section{Lemma 1 [5].}

I. Let $\Phi \in L^{p}([0,2 \pi]), 1<p<\infty$, and $\mathfrak{R}(v)>0$. Denote

$$
\stackrel{\vee}{\Phi}(t):=\sum_{m=n(j)}^{\infty} d_{m}(\Phi) e^{i m t},
$$

where

$$
d_{m}(\Phi):=\int_{0}^{2 \pi} \Phi(\xi) e^{-m v \xi} d \xi, \quad m \geq n(j)
$$

Then $\stackrel{\vee}{\Phi} \in L^{p}([0,2 \pi])$, and $\|\stackrel{\Phi}{\Phi}\| \leq$ const $\cdot\|\Phi\|$ for some positive constant depending only on $p$.

II. Let $f \in E^{p}(D), 1<p<\infty$. For fixed $1 \leq j \leq N$, the Leont'ev coefficients $\left(\kappa_{f}\left(\lambda_{m}^{(j)}\right)\right)_{m \geq n(j)}$ are the Fourier coefficients of some function $F_{j} \in L^{p}([0,2 \pi])$, and $\left\|F_{j}\right\|_{L^{p}} \leq$ const $\cdot\|f\|_{E^{p}}$.

This result was extended in [6] using the first moduli of continuity. Consider the parametrization $z: \partial D \rightarrow$ $[0, T]$ of $\partial D$ : 


$$
z(u)=a_{j}+\frac{a_{j+1}-a_{j}}{\left|a_{j+1}-a_{j}\right|}\left(u-T_{j-1}\right) \quad \text { for } \quad T_{j-1} \leq u \leq T_{j}, \quad j=1, \ldots, N,
$$

where

$$
T_{0}:=0, \quad T_{j}=\sum_{k=1}^{j}\left|a_{k+1}-a_{k}\right|
$$

and

$$
T:=T_{N}:=\sum_{k=1}^{N}\left|a_{k+1}-a_{k}\right|
$$

For $f \in E^{p}(D)$ and $0<h<2 \pi$, let

$$
\delta_{1}(f, h)_{p}:=\sum_{j=1}^{N}\left\{\left(\int_{0}^{h}\left|f\left(a_{j}+\frac{a_{j+1}-a_{j}}{2 \pi} \theta\right)\right|^{p} d \theta\right)^{1 / p}+\left(\int_{2 \pi-h}^{2 \pi}\left|f\left(a_{j}+\frac{a_{j+1}-a_{j}}{2 \pi} \theta\right)\right|^{p} d \theta\right)^{1 / p}\right\} .
$$

The function $\delta_{1}(f, h)_{p}$ is continuous, nonincreasing, and vanishing as $h \rightarrow 0+$.

Theorem 1 [6]. Let $f \in E^{p}(D), 1<p<\infty$, and let $1 \leq j \leq N$ be fixed. Then the Leont'ev coefficients $\kappa_{f}\left(\lambda_{m}^{(j)}\right), m \geq n(j)$, of $f$ are the Fourier coefficients of some function $F_{j} \in L^{p}([0,2 \pi])$. Furthermore,

$$
\omega_{1}\left(F_{j}, h\right)_{p} \leq \mathrm{const} \cdot\left(\omega_{1}(f \circ z, h)_{p}+\delta_{1}(f, h)_{p}\right) .
$$

The proof can be deduced as a special case of Sec. 4.2. Mel'nik used his results in [6] to prove direct approximation theorems for the first moduli. As we will see in Sec. 3, Theorem 1 can also be proved for moduli of arbitrary order.

\section{Extension to Moduli of Arbitrary Order}

To extend Theorem 1, we have to define moduli of smoothness of order $k$ for functions $f \in E^{p}(D)$. This can be done by using the best approximation by algebraic polynomials.

Let $f \in E^{p}(\partial D)$ and let $I \subset \partial D$ be an arc. For $k \in \mathbb{N}_{0}$, the equation

$$
E_{k}(f, I)=\inf _{P_{k}}\left\|f-P_{k}\right\|_{L^{p}(I)}
$$

defines the algebraic best approximation on the arc $I$. Here, the infimum is taken over all algebraic polynomials $P_{k}$ of degree at most $k$. The modulus of order $k$ is defined as follows: 
Definition 1. Let $f \in E^{p}(D), 1<p<\infty$. For $h>0$, consider all partitions

$$
\partial D=\bigcup_{j=1}^{n} I_{j}
$$

where $h / 2 \leq\left|I_{j}\right| \leq h$. The kth metrical modulus of smoothness of the function $f$ is defined as follows:

$$
\omega_{k}(f, h)_{p}:=\omega_{k, \bar{D}}(f, h)_{p}:=\sup \left(\sum_{j=1}^{n} \inf _{P_{k}}\left\|f-P_{k}\right\|_{L^{p}\left(I_{j}\right)}\right)=\sup \left(\sum_{j=1}^{n} E_{k}\left(f, I_{j}\right)\right) .
$$

Here, the supremum is taken over all such partitions.

One can show that these moduli are equivalent to usual moduli of smoothness defined on finite intervals [7]. We can formulate Theorem 1 for the $k$ th moduli.

Theorem 2. Let $f \in E^{p}(D), 1<p<\infty$, and let $1 \leq j \leq N$ be fixed. Then the Leont'ev coefficients $\kappa_{f}\left(\lambda_{m}^{(j)}\right), m \geq n(j)$, are the Fourier coefficients of some function $F_{j} \in L^{p}([0,2 \pi])$ :

$$
\kappa_{f}\left(\lambda_{m}^{(j)}\right)=\frac{1}{2 \pi} \int_{0}^{2 \pi} F_{j}(\theta) e^{i m \theta} d \theta=: c_{m}\left(F_{j}\right)
$$

The kth modulus of $F_{j}$ can be estimated as follows:

$$
\omega_{k}\left(F_{j}, h\right)_{p} \leq \mathrm{const} \cdot\left(\omega_{k}(f, h)_{p}+\delta_{k}(f, h)_{p}\right)
$$

where

$$
\delta_{k}(f, h)_{p}:=\sum_{j=1}^{N} \sum_{n=1}^{k}\left(\begin{array}{l}
k \\
n
\end{array}\right)\left\{\left(\int_{0}^{n h}\left|f\left(a_{j}-\frac{a_{j+1}-a_{j}}{2 \pi} \theta\right)\right|^{p} d \theta\right)^{1 / p}+\left(\int_{2 \pi-n h}^{2 \pi}\left|f\left(a_{j}-\frac{a_{j+1}-a_{j}}{2 \pi} \theta\right)\right|^{p} d \theta\right)^{1 / p}\right\} .
$$

The function $\delta_{k}(f, h)_{p}$ is continuous and nonincreasing for $0<h<2 \pi / h$ and satisfies the relation $\lim _{h \rightarrow 0+} \delta_{k}(f, h)_{p}=0$.

This result enables us to transform the Leont'ev coefficients (2) in the Dirichlet series (1) into the Fourier coefficients of certain functions $F$. Since Theorem 2 provides information on the regularity of $F$, classical Bernstein theorems can be applied to the corresponding Fourier series. This can be used to prove new results on the rate of approximation of the Dirichlet series (1).

The term $\delta_{k}(f, h)_{p}$ cannot be omitted from the theorem, as the following example shows: Let $p=2$ and $f(z)=1$. Suppose that $L(0)=1$. Then $\omega_{k}(f, h)_{2} \equiv 0$, whereas $\delta_{k}(f, h)_{2}=O(\sqrt{h})$ for $h \rightarrow 0$ and all $k \in \mathbb{N}$. For the Leont'ev coefficients, we have 


$$
\kappa_{f}\left(\lambda_{m}^{(j)}\right)=-\frac{1}{\lambda_{m}^{(j)}}=O\left(\frac{1}{m}\right) \quad \text { as } \quad m \rightarrow \infty
$$

We know from Lemma 1 that

$$
F_{j}=\sum_{m \geq n(j)} \kappa_{f}\left(\lambda_{m}^{(j)}\right) e^{i m \cdot} \in L^{2}([0,2 \pi])
$$

The Bernstein theorem [4] yields $\omega_{k}\left(F_{j}, h\right)_{2}=O(\sqrt{h})$ since the approximation with the partial series

$$
S_{n}\left(F_{j}\right)=\sum_{m \geq n(j)} \kappa_{f}\left(\lambda_{m}^{(j)}\right) e^{i m}
$$

gives

$$
\left\|F_{j}-S_{n}\left(F_{j}\right)\right\|_{2}=\left\|\sum_{m>n>n(j)}^{\infty} \frac{1}{\lambda_{m}^{(j)}} e^{i m} .\right\|=\left(\sum_{m>n>n(j)}^{\infty}\left|\frac{1}{\lambda_{m}^{(j)}}\right|^{2}\right)^{1 / 2}=O\left(\frac{1}{\sqrt{n}}\right) \quad \text { as } n \rightarrow \infty .
$$

Thus, the term $\delta_{k}(f, h)_{2}$ is necessary in (3) (see also [6]).

\section{Proof of Theorem 2}

4.1. Preliminaries. Let us first take a closer look at the quasipolynomial

$$
L(z)=\sum_{k=1}^{N} d_{k} e^{a_{k} z}
$$

where $d_{k} \in \mathbb{C} \backslash\{0\}$ and $a_{k}, k=1, \ldots, N$, are the vertices of $D$. Let $\Lambda=\left(\lambda_{m}\right)_{m \in \mathbb{N}}$ be the sequence of its zeros. The entire function $L$ has the following properties [1] (Chap. 1, Sec. 2):

I. For sufficient large $C$, the zeros $\lambda_{n}^{(j)}$ of $L$ such that $\left|\lambda_{n}^{(j)}\right|>C$ are of the form $\lambda_{n}^{(j)}=\tilde{\lambda}_{n}^{(j)}+\delta_{n}^{(j)}$, where

$$
\tilde{\lambda}_{n}^{(j)}=\frac{2 \pi n i}{a_{j+1}-a_{j}}+q_{j} e^{i \beta_{j}}
$$

and $\left|\delta_{n}^{(j)}\right| \leq e^{-a n}$. Here, $0<a=$ const, $j=1, \ldots, N, n>n_{0}$, and $a_{N+1}:=a_{1}$. The parameters $b_{j}$ and $q_{j}$ are defined by the formula

$$
e^{q_{j}\left(a_{j+1}-a_{j}\right) e^{i \beta_{j}}}=-\frac{d_{j}}{d_{j+1}}
$$


where $d_{N+1}:=d_{1}$. Hence, the zeros $\lambda_{n}^{(j)}$ are simple. The set of zeros $\Lambda$ can be represented in the form

$$
\Lambda=\left\{\lambda_{n}\right\}_{n=1, \ldots, n_{0}} \cup\left(\bigcup_{j=1}^{N}\left\{\lambda_{n}^{(j)}\right\}_{n=n(j), n(j)+1, \ldots}\right)
$$

II. There are positive constants $A_{1}$ and $c_{1}$ such that, for all $n \geq n(j)$ and $\xi \in\left[a_{j}, a_{k}\right]$, we have

$$
\left|e^{-\lambda_{n}^{(j)}\left(\xi-a_{k}\right)}-e^{-\tilde{\lambda}_{n}^{(j)}\left(\xi-a_{k}\right)}\right| \leq A_{1} e^{-c_{1} n}
$$

Here, $\left[a_{j}, a_{k}\right]$ denotes the line between the vertices $a_{j}$ and $a_{k}$ in the complex plane.

For simplicity, we assume that all zeros of $L$ are simple. We use properties I and II to treat the zeros of $L$ and to estimate the complex exponentials in the Dirichlet series (1). In addition, we need the following result on multipliers:

Theorem 3 (J. Marcinkiewicz, [8], Theorem 4.14). Let $\left(a_{n}\right)_{n \in \mathbb{N}_{0}} \subset \mathbb{C}$ be some series such that

$$
\left|a_{n}\right|<M \quad \text { and } \quad \sum_{j=2^{n}}^{2^{n+1}-1}\left|a_{j}-a_{j+1}\right| \leq M
$$

for all $n \in \mathbb{N}_{0}$ and some suited positive constant $M$. Let

$$
f=\sum_{n=0}^{\infty} c_{n} e^{i n \cdot} \in L^{p}([0,2 \pi]), \quad 1<p<\infty .
$$

Then there exists a function $h \in L^{p}([0,2 \pi])$ with

$$
h=\sum_{n=0}^{\infty} c_{n} a_{n} e^{i n .} \quad \text { and } \quad\|h\| \leq C(p) M\|f\|,
$$

where the constant $C(p)>0$ depends only on $p$.

We now have all means for the proof of Theorem 2.

4.2. Proof. The existence of a function $F_{j} \in L^{p}([0,2 \pi])$ with the indicated properties is shown in assertion II of Lemma 1. Thus, we just have to examine the regularity of $F_{j}$. Using conditions I and II of Sec. 4.1, we can write 


$$
\begin{aligned}
\kappa_{f}\left(\lambda_{n}^{(j)}\right)= & \sum_{k=1}^{N} d_{k} \int_{a_{j}}^{a_{k}} f(z) e^{-\lambda_{n}^{(j)}\left(z-a_{k}\right)} d z=\sum_{k=1}^{N} d_{k} \int_{a_{j}}^{a_{k}} f(z) e^{-\tilde{\lambda}_{n}^{(j)}\left(z-a_{k}\right)} d z+O\left(e^{-c n}\right) \\
= & d_{j+1} \frac{a_{j+1}-a_{j}}{2 \pi} \int_{0}^{2 \pi} f\left(a_{j+1}-\frac{a_{j}-a_{j+1}}{2 \pi} \theta\right) e^{q_{j} e^{i \beta_{j}} \frac{a_{j+1}-a_{j}}{2 \pi} \theta} e^{i n \theta} d \theta \\
& +\sum_{\substack{k=1 \\
k \neq j, j+1}}^{N} d_{k} \frac{a_{k}-a_{j}}{2 \pi} \int_{0}^{2 \pi} f\left(a_{k}+\frac{a_{j}-a_{k}}{2 \pi} \theta\right) e^{q_{j} e^{i \beta_{j}} \frac{a_{k}-a_{j}}{2 \pi} \theta} e^{-n i \frac{a_{j}-a_{k}}{a_{j+1}-a_{j}} \theta} d \theta+O\left(e^{-c n}\right) .
\end{aligned}
$$

The first term is obviously the $n$th Fourier coefficient of some function with modulus of order $\omega_{k, \bar{D}}(f, h)_{p}$. Using assertion I of Lemma 1 for the second term, we just have to analyse the regularity of $\check{\Phi}$ with respect to the regularity of some function $\Phi \in L^{p}([0,2 \pi])$ since

$$
\Re\left(i \frac{a_{j}-a_{k}}{a_{j+1}-a_{j}}\right)>0
$$

Then the required assertion follows from the inequality $\omega_{k}\left(\left.f\right|_{\left[a_{j}, a_{j+1}\right]}, h\right)_{p} \leq \omega_{k}(f, h)_{p}$.

Let $h>0, \Re(v)>0, \alpha \in \mathbb{R}$, and $\Phi \in L^{p}([0,2 \pi])$. We will show that the series of coefficients $\left(d_{m}(\Phi)\right)_{m \geq n(j)}$ is the series of Fourier coefficients of some function $\stackrel{\vee}{\Phi} \in L^{p}([0,2 \pi])$ with

$$
\omega_{k}(\stackrel{\vee}{\Phi}, h)_{p} \leq \text { const } \cdot\left(\omega_{k}(\Phi, h)_{p}+\delta_{k}^{\prime}(\Phi, h)_{p}\right),
$$

where

$$
\delta_{k}^{\prime}(\Phi, h)_{p}:=\sum_{n=1}^{k}\left(\begin{array}{l}
k \\
n
\end{array}\right)\left\{\left(\int_{0}^{n h}|\Phi(u)|^{p} d u\right)^{1 / p}+\left(\int_{2 \pi-n h}^{2 \pi}|\Phi(u)|^{p} d u\right)^{1 / p}\right\} .
$$

Let $\varphi \in L^{p}([0,2 \pi])$. Then

$$
\begin{aligned}
A & :=\int_{0}^{2 \pi}\left(\Delta_{-|\alpha|}^{k} \varphi\right)(u) e^{-m v u} d u-\left(\Delta_{|\alpha|}^{k} e^{-m v \cdot}\right)(0) \int_{0}^{2 \pi} \varphi(u) e^{-m v u} d u \\
& =\sum_{n=0}^{k}(-1)^{k-n}\left(\begin{array}{l}
k \\
n
\end{array}\right)\left\{\int_{0}^{2 \pi} \varphi(u-n|\alpha|) e^{-m v u} d u-\int_{0}^{2 \pi} \varphi(u) e^{-m v(u+n|\alpha|)} d u\right\} \\
& =\sum_{n=0}^{k}(-1)^{k-n}\left(\begin{array}{l}
k \\
n
\end{array}\right)\left\{\int_{0}^{2 \pi} \varphi(u-n|\alpha|) e^{-m v u} d u-\int_{n|\alpha|}^{2 \pi+n|\alpha|} \varphi(u-n|\alpha|) e^{-m v u} d u\right\}
\end{aligned}
$$




$$
\begin{aligned}
& =\sum_{n=0}^{k}(-1)^{k-n}\left(\begin{array}{l}
k \\
n
\end{array}\right)\left\{\int_{0}^{n|\alpha|} \varphi(u-n|\alpha|) e^{-m v u} d u-\int_{2 \pi}^{2 \pi+n|\alpha|} \varphi(u-n|\alpha|) e^{-m v u} d u\right\} \\
& =\left(1-e^{-2 \pi m v}\right) \sum_{n=0}^{k}(-1)^{k-n}\left(\begin{array}{l}
k \\
n
\end{array}\right) \int_{0}^{n|\alpha|} \varphi(u-n|\alpha|) e^{-m v u} d u .
\end{aligned}
$$

With the notation of assertion I of Lemma 1, we have

$$
\begin{aligned}
\Delta_{\alpha}^{k} \check{\varphi}(t) & =\sum_{m=n(j)}^{\infty} d_{m}(\varphi)\left(\Delta_{\alpha}^{k} e^{i m \cdot}\right)(t)=\sum_{m=n(j)}^{\infty} d_{m}(\varphi)\left(\Delta_{\alpha}^{k} e^{i m \cdot}\right)(0) e^{i m t} \\
& =\sum_{m=n(j)}^{\infty} \int_{0}^{2 \pi} \varphi(\xi) e^{-m v \xi} d \xi\left(\Delta_{\alpha}^{k} e^{i m \cdot}\right)(0) e^{i m t} \\
& =\sum_{m=n(j)}^{\infty}\left(\int_{0}^{2 \pi}\left(-\Delta_{-|\alpha|}^{k} \varphi\right)(u) e^{-m v u} d u+A\right) \frac{\left(\Delta_{\alpha}^{k} e^{i m \cdot}\right)(0)}{\left(-\Delta_{|\alpha|}^{k} e^{-m v \cdot}\right)(0)} e^{i m t} \\
& =\sum_{m=n(j)}^{\infty} d_{m}\left(-\Delta_{-|\alpha|}^{k} \varphi\right) \frac{\left(\Delta_{\alpha}^{k} e^{i m \cdot}\right)(0)}{\left(-\Delta_{|\alpha|}^{k} e^{-m v \cdot}\right)(0)} e^{i m t} \\
& +\sum_{m=n(j)}^{\infty} \sum_{n=0}^{k}(-1)^{k-n}\left(\begin{array}{l}
k \\
n
\end{array}\right)_{0}^{n|\alpha|} \varphi(u-n|\alpha|) e^{-m v u} d u\left(1-e^{-2 \pi v m}\right) \frac{\left(\Delta_{\alpha}^{k} e^{i m \cdot}\right)(0)}{\left(-\Delta_{|\alpha|}^{k} e^{-m v \cdot}\right)(0)} e^{i m t} \\
& =\sum_{m=n(j)}^{\infty} d_{m}\left(-\Delta_{-|\alpha|}^{k} \varphi\right) \mu_{m} e^{i m t}+\sum_{m=n(j)}^{\infty} \sum_{n=0}^{k}(-1)^{k-n}\left(\begin{array}{l}
k \\
n
\end{array}\right)_{0}^{n|\alpha|} \varphi(u-n|\alpha|) e^{-m v u} d u \tilde{\mu}_{m} e^{i m t},
\end{aligned}
$$

where

$$
\mu_{m}=\frac{\left(\Delta_{\alpha}^{k} e^{i m \cdot}\right)(0)}{\left(-\Delta_{|\alpha|}^{k} e^{-m v \cdot}\right)(0)} \quad \text { and } \quad \tilde{\mu}_{m}=\left(1-e^{-2 \pi m v}\right) \mu_{m}
$$

We will show that $\mu_{m}$ and $\tilde{\mu}_{m}$ are multipliers in $L^{p}([0,2 \pi])$. Thus, the Fourier series weighted with $\left(\mu_{m}\right)_{m \geq n(j)}$ and $\left(\tilde{\mu}_{m}\right)_{m \geq n(j)}$ both converge in $L^{p}([0,2 \pi])$. First, consider $\mu_{m}$. We have

$$
\mu_{m+1}-\mu_{m}=\int_{m}^{m+1}\left(\frac{d}{d x} \frac{\left(\Delta_{\alpha}^{k} e^{i x \cdot}\right)(0)}{\left(-\Delta_{|\alpha|}^{k} e^{-x v \cdot}\right)(0)}\right) d x
$$

and, furthermore, 


$$
\frac{d}{d x} \frac{\left(\Delta_{\alpha}^{k} e^{i x \cdot}\right)(0)}{\left(-\Delta_{|\alpha|}^{k} e^{-x v \cdot}\right)(0)}=-\frac{d}{d x} \frac{\left(1-e^{i x \alpha}\right)^{k}}{\left(1-e^{-x v|\alpha|}\right)^{k}}
$$

Let $\varepsilon>0$ be fixed and let $|\alpha|<\min \{\Re(v), 1\}$. We split the weighted Fourier series in two parts.

First, let $m|\alpha|<\varepsilon$. For $m \leq x \leq m+1$, we have

$$
\left|\frac{d}{d x} \frac{\left(\Delta_{\alpha}^{k} e^{i x \cdot}\right)(0)}{\left(-\Delta_{|\alpha|}^{k} e^{-x v}\right)(0)}\right|=\left|\frac{d}{d x} \frac{\left(1-e^{i x \alpha}\right)^{k}}{\left(1-e^{-x v|\alpha|}\right)^{k}}\right|=k\left|\frac{\left(1-e^{i x \alpha}\right)^{k-1}}{\left(1-e^{-x v|\alpha|}\right)^{k-1}}\right|\left|\frac{d}{d x}\left(\frac{1-e^{i x \alpha}}{1-e^{-x v|\alpha|}}\right)\right| .
$$

We investigate how this term behaves as $|\alpha| \rightarrow 0$ because, for $|\alpha|>\gamma$ with some $\gamma>0$, the term is bounded in the domain $m|\alpha|<\varepsilon$ for continuity reasons. For $k=1$, it is easily seen that

$$
\left|\frac{d}{d x}\left(\frac{1-e^{i x \alpha}}{1-e^{-x v|\alpha|}}\right)\right|=|\alpha| \frac{-i\left(1-e^{-x v|\alpha|}\right) e^{i x \alpha}-v \operatorname{sign}(\alpha)\left(1-e^{i x \alpha}\right) e^{-x v|\alpha|}}{\left(1-e^{-x v|\alpha|}\right)^{2}} \mid .
$$

The second term converges to $\left|\frac{1-i v \operatorname{sign}(\alpha)}{2 v}\right|$ if $|\alpha| \rightarrow 0$. Since $m \leq x \leq m+1$ and $m|\alpha|<\varepsilon$, we can estimate the whole term by some constant independent of $\alpha$ and $x$. Hence, $\left|\mu_{m}\right| \leq$ const for $m|\alpha|<\varepsilon$ and some constant independent of $\alpha$. By induction and Eq. (6), we get

$$
\left|\frac{d}{d x} \frac{\left(\Delta_{\alpha}^{k} e^{i x \cdot}\right)(0)}{\left(-\Delta_{|\alpha|}^{k} e^{-x v}\right)(0)}\right| \leq \text { const } \cdot|\alpha|
$$

where the constant does not depend on $x$ and $\alpha$. Furthermore, $\left|\mu_{m}\right|<$ const for some constant independent of $\alpha$. Using Eq. (5), we deduce

$$
\left|\mu_{m+1}-\mu_{m}\right| \leq \mathrm{const} \int_{m}^{m+1}|\alpha| d x=\text { const } \cdot|\alpha|,
$$

and, thus,

$$
\sum_{m<\varepsilon /|\alpha|}\left|\mu_{m+1}-\mu_{m}\right| \leq \text { const. }
$$

For $\Phi \in L^{p}([0,2 \pi])$ and $\stackrel{v}{\Phi}$ as in Lemma 1, by using Theorem 3, we conclude that

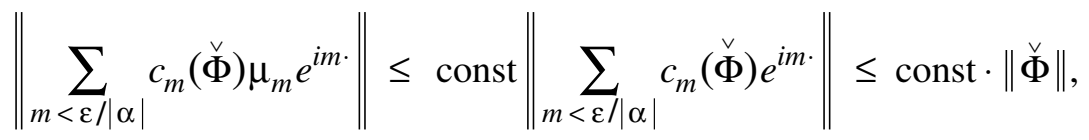

since $\left(c_{m}(\stackrel{\vee}{\Phi})\right)_{m \in \mathbb{Z}}$ is the sequence of Fourier coefficients of $\stackrel{\vee}{\Phi}$. 
Second, let $m|\alpha| \geq \varepsilon$. We define

$$
\mu_{m}^{\prime}:=\frac{1}{\left(1-e^{-m v|\alpha|}\right)^{k}}
$$

and deduce

$$
\begin{aligned}
& \left|\mu_{m+1}^{\prime}-\mu_{m}^{\prime}\right| \leq\left|\frac{\left(1-e^{-m v|\alpha|}\right)^{k}-\left(1-e^{-(m+1) v|\alpha|}\right)^{k}}{\left(1-e^{-m v|\alpha|}\right)^{k}\left(1-e^{-(m+1) v|\alpha|}\right)^{k}}\right| \\
& =\left|\frac{\sum_{n=1}^{k}(-1)^{k-n}\left(\begin{array}{l}
k \\
n
\end{array}\right) e^{-m n v|\alpha|}\left(1-e^{-n v|\alpha|}\right)}{\left(1-e^{-m v|\alpha|}\right)^{k}\left(1-e^{-(m+1) v|\alpha|}\right)^{k}}\right| \\
& \leq e^{-m \Re(v)|\alpha|}\left|\frac{\sum_{n=1}^{k}(-1)^{k-n}\left(\begin{array}{l}
k \\
n
\end{array}\right)\left(1-e^{-n v|\alpha|}\right)}{\left(1-e^{-m v|\alpha|}\right)^{k}\left(1-e^{-(m+1) v|\alpha|}\right)^{k}}\right| \\
& \leq e^{-m \Re(v)|\alpha|}|\alpha|\left|\frac{\sum_{n=1}^{k}(-1)^{k-n}\left(\begin{array}{l}
k \\
n
\end{array}\right) \frac{1-e^{-n v|\alpha|}}{|\alpha|}}{\left(1-e^{-m v|\alpha|}\right)^{k}\left(1-e^{-(m+1) v|\alpha|}\right)^{k}}\right| \\
& \leq e^{-m \Re(v)|\alpha|}|\alpha|\left|\frac{C(k, v)}{\left(1-e^{-m v|\alpha|}\right)^{k}\left(1-e^{-(m+1) v|\alpha|}\right)^{k}}\right| \leq C(k, v, \varepsilon)|\alpha| e^{-m \Re(v)|\alpha|}
\end{aligned}
$$

for positive constants $C(k, v)$ and $C(k, v, \varepsilon)$. Thus, since $|\alpha|<\min \{\Re(v), 1\}$, we have

$$
\begin{aligned}
\sum_{m \geq \varepsilon /|\alpha|}\left|\mu_{m+1}^{\prime}-\mu_{m}^{\prime}\right| & \leq \sum_{l=0}^{\infty}\left|\mu_{[\varepsilon /|\alpha|]+l+1}^{\prime}-\mu_{[\varepsilon /|\alpha|]+l}^{\prime}\right| \\
& \leq C(k, v, \varepsilon)|\alpha| \sum_{l=0}^{\infty} e^{-([\varepsilon /|\alpha|]+l) \Re(v)|\alpha|} \\
& \leq C(k, v, \varepsilon)|\alpha| \frac{1}{1-e^{-\Re(v)|\alpha|}} e^{-[\varepsilon /|\alpha|] \Re(v)|\alpha|} \\
& \leq C(k, v, \varepsilon)|\alpha| \frac{1}{1-e^{-\Re(v)|\alpha|}} \leq C(k, v, \varepsilon) \Re(v) .
\end{aligned}
$$


Obviously, $\left|\mu_{m}^{\prime}\right| \leq C_{\varepsilon}$ for some positive constant $C_{\varepsilon}$ depending only on $\varepsilon$. Hence, for $\Phi \in L^{p}([0,2 \pi])$ with $\stackrel{\vee}{\Phi}$ as in Lemma 1 , by using Theorem 3 we get

$$
\left\|\sum_{m \geq \varepsilon|\alpha|^{-1}} c_{m}(\stackrel{v}{\Phi}) \mu_{m}^{\prime} e^{i m} \cdot\right\| \leq \text { const } \cdot\left\|\sum_{m \geq \varepsilon|\alpha|^{-1}} c_{m}(\stackrel{v}{\Phi}) e^{i m} \cdot\right\| \leq \text { const } \cdot\|\stackrel{v}{\Phi}\| .
$$

Denote

$$
\stackrel{\vee}{\Phi}_{\varepsilon}(t):=\sum_{m \geq \varepsilon|\alpha|^{-1}} c_{m}(\stackrel{\vee}{\Phi}) \mu_{m}^{\prime} e^{i m t}
$$

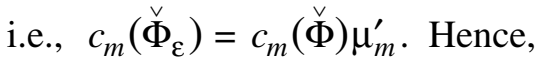

$$
\begin{aligned}
& \left\|\sum_{m \geq \varepsilon|\alpha|^{-1}} c_{m}(\stackrel{v}{\Phi}) \mu_{m} e^{i m \cdot}\right\|=\left\|\sum_{m \geq \varepsilon|\alpha|^{-1}} c_{m}(\stackrel{v}{\Phi}) \mu_{m}^{\prime}\left(1-e^{i m \alpha}\right)^{k} e^{i m} \cdot\right\| \\
& =\left\|\sum_{m \geq \varepsilon|\alpha|^{-1}} c_{m}\left(\stackrel{\check{\Phi}}{\varepsilon}_{\varepsilon}\right)\left(1-e^{i m \alpha}\right)^{k} e^{i m} \cdot\right\|=\left\|\sum_{m \geq \varepsilon|\alpha|^{-1}} c_{m}\left(\Delta_{-\alpha}^{k} \stackrel{v}{\Phi}_{\varepsilon}\right) e^{i m \cdot}\right\| \\
& \leq\left\|\Delta_{-\alpha}^{k} \stackrel{\vee}{\Phi}_{\varepsilon}\right\| \leq(k+1)\left\|\stackrel{\vee}{\Phi}_{\varepsilon}\right\| \leq \text { const } \cdot\|\stackrel{\vee}{\Phi}\|,
\end{aligned}
$$

where we have used (8). Using (7) and (9), we can deduce

$$
\left\|\sum_{m=n(j)}^{\infty} c_{m}(\check{\Phi}) \mu_{m} e^{i m \cdot}\right\|=\left\|\sum_{n(j) \leq m<\varepsilon|\alpha|^{-1}} c_{m}(\stackrel{\check{\Phi}}{\Phi}) \mu_{m} e^{i m \cdot}\right\|+\left\|\sum_{m \geq \varepsilon|\alpha|^{-1}} c_{m}(\check{\Phi}) \mu_{m} e^{i m \cdot}\right\| \leq \text { const } \cdot\|\check{\Phi}\| .
$$

Hence, $\mu_{m}$ is a multiplier in $L^{p}([0,2 \pi])$.

The same can be shown for $\tilde{\mu}_{m}$. We have

$$
\begin{aligned}
\left|\tilde{\mu}_{m+1}-\tilde{\mu}_{m}\right| & \leq\left|\left(1-e^{-2 \pi(m+1) v}\right) \mu_{m+1}-\left(1-e^{-2 \pi m v}\right) \mu_{m}\right| \\
& \leq\left|\mu_{m+1}-\mu_{m}\right|\left(1+e^{-2 \pi m \Re(v)}\right)+\left|\mu_{m+1}\right|\left|e^{-2 \pi m v}-e^{-2 \pi(m+1) v}\right| \\
& \leq\left|\mu_{m+1}-\mu_{m}\right|\left(1+e^{-2 \pi m \Re(v)}\right)+\left|\mu_{m+1}\right| e^{-2 \pi m \Re(v)}\left|1-e^{-2 \pi v}\right| \\
& \leq \text { const } \cdot\left(\left|\mu_{m+1}-\mu_{m}\right|+e^{-a m}\right),
\end{aligned}
$$

for some positive constant $a>0$. Thus, $\tilde{\mu}_{m}$ is a multiplier in $L^{p}([0,2 \pi])$, too. We have 


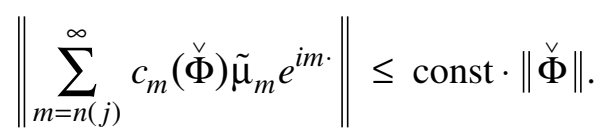

For $|\alpha|<h$, using relations (4) and Lemma 1 we get

$$
\begin{aligned}
& \left\|\Delta_{\alpha}^{k} \stackrel{v}{\Phi}\right\| \leq\left\|\sum_{m=n(j)}^{\infty} d_{m}\left(-\Delta_{-|\alpha|}^{k} \Phi\right) \mu_{m} e^{i m \cdot}\right\|+\left\|\sum_{m=n(j)}^{\infty} \sum_{n=0}^{k}(-1)^{k-n}\left(\begin{array}{l}
k \\
n
\end{array}\right)_{0}^{n|\alpha|} \Phi(u-n|\alpha|) e^{-m v u} d u \tilde{\mu}_{m} e^{i m \cdot}\right\| \\
& \leq \text { const } \cdot\left\|\sum_{m=n(j)}^{\infty} d_{m}\left(-\Delta_{-|\alpha|}^{k} \Phi\right) e^{i m} \cdot\right\|+\text { const } \cdot\left\|\sum_{m=n(j)}^{\infty} \sum_{n=0}^{k}(-1)^{k-n}\left(\begin{array}{l}
k \\
n
\end{array}\right) \int_{0}^{n|\alpha|} \Phi(u-n|\alpha|) e^{-m v u} d u e^{i m} \cdot\right\| \\
& \leq \text { const } \cdot\left\|\Delta_{-|\alpha|}^{k} \Phi\right\|+\text { const } \cdot\left\|\sum_{m=n(j)}^{\infty} \sum_{n=0}^{k}(-1)^{k-n}\left({ }_{n}^{k}\right)_{0}^{2 \pi}\left(\chi_{[0, n|\alpha|]}(u) \Phi(u-n|\alpha|)\right) e^{-m v u} d u e^{i m \cdot}\right\| \\
& =\text { const } \cdot\left(\left\|\Delta_{-|\alpha|}^{k} \Phi\right\|+\left\|\sum_{m=n(j)}^{\infty} \sum_{n=0}^{k}(-1)^{k-n}\left(\begin{array}{l}
k \\
n
\end{array}\right) d_{m}\left(\chi_{[0, n|\alpha|]}\right) \Phi(\cdot-n|\alpha|) e^{i m \cdot}\right\|\right) \\
& \leq \text { const } \cdot\left(\left\|\Delta_{-|\alpha|}^{k} \Phi\right\|+\left\|\sum_{n=1}^{k}(-1)^{k-n}\left(\begin{array}{l}
k \\
n
\end{array}\right) \chi_{[0, n|\alpha|]} \Phi(\cdot-n|\alpha|)\right\|\right) \\
& \leq \text { const } \cdot\left(\omega_{k}(\Phi, h)_{p}+\sum_{n=1}^{k}\left(\begin{array}{l}
k \\
n
\end{array}\right)\left(\int_{0}^{n|\alpha|}|\Phi(u-n|\alpha|)|^{p} d u\right)^{1 / p}\right) \\
& \leq \text { const } \cdot\left(\omega_{k}(\Phi, h)_{p}+\delta_{k}^{\prime}(\Phi, h)_{p}\right),
\end{aligned}
$$

where $\chi_{[a, b]}$ denotes the characteristic function of the interval $[a, b]$. Passing to the supremum, we get

$$
\omega_{k}(\stackrel{\vee}{\Phi}, h)_{p}=\sup _{0<|\alpha|<h}\left\|\Delta_{\alpha}^{k} \Phi\right\|_{p} \leq \text { const } \cdot\left(\omega_{k}(\Phi, h)_{p}+\delta_{k}^{\prime}(\Phi, h)_{p}\right)
$$

and the assertion is proved.

The author wants to express her gratitude to V. V. Andrievskii and R. Lasser for many valuable comments on the subject.

This work was supported by the "Deutsche Forschungsgemeinschaft" through the graduate program “Angewandte Algorithmische Mathematik," Technische Universität München. 


\section{REFERENCES}

1. A. F. Leont'ev, Exponential Series [in Russian], Nauka, Moscow (1976).

2. B. Ja. Lewin and Ju. I. Ljubarskii, "Interpolation by means of special classes of entire functions and related expansions in series of exponentials," Math. USSR Izv., 9, 621-662 (1975).

3. A. M. Sedletskii, "Bases of exponential functions in the space $E^{p}$ on convex polygons," Math. USSR Izv., 13(2), 387-404 (1979).

4. N. I. Achieser, Theory of Approximation, Dover, New York (1992).

5. Yu. I. Mel'nik, "Some properties of exponential series representing functions being regular in convex polygons," in: Some Problems in the Theory of Approximation of Functions [in Russian], Kiev (1985), pp. 69-81.

6. Yu. I. Mel'nik, "Direct theorems for the approximation of functions regular in convex polygons by exponential polynomials in the integral metric," Ukr. Mat. Zh., 40, 498-504 (1988).

7. Yu. A. Brudnyi, "Piecewise polynomial approximation, embedding theorem and rational approximation," Lect. Notes Math., 556, 73-98 (1976).

8. A. Zygmund, Trigonometric Series, Cambridge Univ. Press (1988). 